IdeAs

Idées d'Amériques

15 | 2020

Eau et gestion de l'eau dans les Amériques

\title{
La néolibéralisation de l'économie chilienne dans une perspective historique
}

Manuel Gárate

\section{OpenEdition}

Journals

Édition électronique

URL : http://journals.openedition.org/ideas/8344

DOI : 10.4000/ideas.8344

ISSN : 1950-570

Éditeur

Institut des Amériques

Référence électronique

Manuel Gárate, "La néolibéralisation de l'économie chilienne dans une perspective historique », IdeAs [En ligne], 15 | 2020, mis en ligne le 01 mars 2020, consulté le 25 mars 2020. URL : http:// journals.openedition.org/ideas/8344 ; DOI : https://doi.org/10.4000/ideas.8344

Ce document a été généré automatiquement le 25 mars 2020

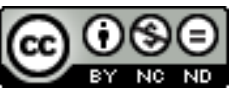

IdeAs - Idées d'Amériques est mis à disposition selon les termes de la licence Creative Commons Attribution - Pas d'Utilisation Commerciale - Pas de Modification 4.0 International. 


\title{
La néolibéralisation de l'économie chilienne dans une perspective historique
}

\author{
Manuel Gárate
}

1 Les événements qui ont eu lieu au Chili depuis le 18 octobre 2019 ont été définis, notamment par la presse chilienne et la classe politique, comme une «explosion sociale ", faisant référence à un genre de protestation plutôt inattendue et spontanée. Cependant, l'ampleur des mobilisations, leur force et le fait qu'elles se soient produites sur l'ensemble du territoire, suggèrent plutôt une sorte de soulèvement populaire ou d'insurrection avec des caractéristiques beaucoup plus profondes et liées à d'autres mouvements sociaux similaires de l'histoire du $\mathrm{Xx}^{\mathrm{e}}$ siècle chilienne (Milos P., 2007 : 19-20). Bien que les événements qui ont déclenché ce processus aient pour origine la hausse du prix du ticket de métro et la réaction des lycéens avec des évasions massives du transport public dans la capitale, une série d'autres revendications politiques et sociales a été mise en évidence au cours des semaines qui ont suivi le 18 octobre. Cellesci sont liées au modèle de développement que le pays maintient depuis 45 ans au moins, et qui se cristallise également dans la demande d'une nouvelle constitution politique.

2 Le slogan répété “ce n'est pas contre 30 pesos mais contre 30 ans" traduit précisément le fait que pour une partie importante de la population chilienne, le retour à la démocratie politique, depuis 1990, n'a pas entraîné de changement substantiel de leurs conditions de vie, et ce malgré une baisse de l'extrême pauvreté et une augmentation significative du revenu des foyers. Le problème de la marginalité, des inégalités économiques et de l'accès aux services reste un sujet que l'élite politique et économique (tant du centregauche que de la droite) a minimisé pour privilégier la croissance économique et le ciblage des politiques sociales envers les plus pauvres. Le principe de subsidiarité de l'État et la prépondérance des politiques assistencialistes ont engendré une importante ségrégation économique dans la population, qui prend la forme, par exemple, d'énormes cercles de marginalité dans de grandes villes extrêmement étendues et laissées au libre jeu du marché immobilier et de la spéculation du sol. Mais ce n'est là 
qu'un des éléments de la ségrégation sociale et de la privatisation de la vie sociale qui se sont manifestés dans le cycle de protestations du 2019-2020. Pour tenter d'expliquer l'origine du phénomène, il est nécessaire de faire une brève histoire du modèle économique chilien et de sa consolidation ultérieure pendant la transition démocratique.

\section{L'origine du modèle économique néolibéral}

3 La transformation majeure du modèle de développement chilien a eu lieu au cours de la période 1975-1986, en abandonnant ce qui avait été jusque-là une expérience hétérodoxe de construction d'un État social et moteur de l'industrialisation. Ce dernier était sorti de la crise du modèle d'exportation des matières premières hérité du $\mathrm{XIX}^{\mathrm{e}}$ siècle, qui fut fortement affecté par la Première Guerre mondiale puis par la Grande Dépression de 1929-30 (Palma G., 1984: 62). Bien que le modèle chilien d'industrialisation par substitution des importations ait montré ses limites vers le milieu des années 1950, il avait donné naissance à une nouvelle classe moyenne liée à l'État et à la massification de l'enseignement public. À cela s'ajoutait une classe ouvrière issue de l'industrie nationale, représentée par des syndicats et différents partis politiques dont un nouveau centre social-chrétien (Démocratie chrétienne), tout comme une gauche plus traditionnelle (Parti socialiste et Parti communiste, principalement). L'approfondissement de ce modèle développementaliste (bien que très dépendant de l'exportation du cuivre) et sa transformation en une économie socialiste, étaient au cœur de la promesse électorale de Salvador Allende (1970-1973).

4 Le coup d'État de septembre 1973 a signifié la fin tragique du projet socialiste chilien, mais pas entièrement de l'État développementaliste qui avait émergé depuis les années 1930. Ce n'est qu'en 1975 qu'un groupe de jeunes économistes et technocrates diplômés aux États-Unis, appelés les Chicago Boys, a repris complètement en charge l'économie chilienne. Cela a provoqué un tournant important dans la politique économique de la dictature militaire, car jusqu'à ce moment-là la vision nationaliste-développementaliste de plusieurs hauts officiers militaires consistait à consolider un État fort (protectionniste) et à négocier avec les syndicats à condition que ceux-ci restent loin des partis de gauche. Mais ce groupe d'officiers a été vaincu en interne par ceux qui ont réussi à imposer au sein de la Junte militaire l'idée d'un changement radical du modèle de développement économique (Valdivia V., 2003 : 151). Cela devait s'accompagner d'un système institutionnel qui consoliderait ces changements socio-économiques dans un futur retour à la démocratie. C'est ce qu'on a appelé la fusion "Chicago-grémialiste ", qui combine le conservatisme politique, le libéralisme économique radical et une profonde méfiance envers la démocratie et la politique. Cette association de civils avec les militaires a favorisé le général Pinochet lui-même, qui a cherché à renforcer son pouvoir au sein de la Junte et à éliminer ainsi toute dissidence. Ce processus s'est consolidé au cours de la période 1975-1980, qui coïncide avec la promulgation de la nouvelle Constitution de 1980, en vigueur jusqu'à aujourd'hui malgré ses nombreuses réformes ultérieures.

5 La néolibéralisation de l'économie chilienne a commencé avec le grand ajustement du secteur public en 1975-1976 (thérapie de choc), lorsque la taille de l'État a été considérablement réduite, ce qui a généré un chômage élevé et de la précarité de l'emploi, dans un contexte de forte répression de l'État contre les principaux partis de 
gauche et les organisations syndicales (Gárate M., 2012 : 196). Par la suite, à partir de 1979, les technocrates du régime se sont tournés vers ce qu'ils ont appelé les grandes modernisations ou transformations structurelles de l'économie chilienne. Il s'agit notamment de la privatisation partielle ou totale des principaux services sociaux: santé, éducation et retraites, laissant à l'état un rôle purement subsidiaire. Cela a aussi concerné les grandes entreprises publiques créées depuis 1940 et la réforme du travail qui a libéralisé les relations entre travailleurs et patronat, diminuant considérablement la capacité d'action des syndicats et des associations professionnelles. A cela, il faut ajouter une profonde réforme des échanges commerciaux, mise en œuvre par la baisse drastique des tarifs et l'ouverture sans restrictions aux importations; tout cela a contribué à la libéralisation du marché financier et à un endettement extérieur élevé.

6 Ces politiques publiques ont généré une brève période de boom économique (1978-1981), fondé sur l'endettement extérieur et un prix du dollar artificiellement bas. Le pays a été envahi par des produits importés bon marché, tandis que l'industrie nationale agonisait et le chômage de masse apparaissait (Gárate M., 2013: 286). La grave crise économique de 1983-1986 a provoqué la quasi-faillite du système bancaire chilien, qui n'a pu survivre que grâce à des subventions directes de l'État et au sauvetage de nombreuses entreprises privées. La crise a été le déclencheur d'un long cycle de protestations qui démontra la forte précarité de la vie des chiliens et l'action minimale de l'État en matière de protection sociale. Ce cycle de mobilisations a ouvert la voie à l'union de l'opposition contre la dictature militaire.

\section{L'héritage économique de la dictature}

7 Le régime autoritaire a réussi à résister à la pression sociale, mais Pinochet a dû quitter la tête de l'État chilien en 1990 après avoir perdu le référendum de 1988. C'est ainsi que la transition démocratique chilienne a commencé, dans un contexte où l'armée avait un énorme pouvoir de facto, également soutenue par les partis de droite et le grand patronat. Les forces démocratiques (la Concertación) ont été contraintes dès le premier jour de négocier leur projet de gouvernement et, surtout, d'accepter les conditions de la Constitution de 1980 (voir article d'Escudero et Gajardo dans ce dossier). Cette dernière assurait le maintien des piliers du modèle économique néolibéral et le pouvoir du veto des militaires par le biais de sénateurs désignés et de l'existence de quorums parlementaires ultra majoritaires $(4 / 7$ ou $2 / 3)$ pour empêcher toute réforme importante du modèle économique.

8 Bien que la Concertación ait réussi à donner un certaine orientation sociale aux politiques économiques, notamment sous le gouvernement de Patricio Aylwin (1990-1993), les fondements du modèle ont surtout été renforcés: la santé et les retraites sont restés entre les mains d'entreprises privées (ISAPRES et AFP'), la politique d'exportation des matières premières a été approfondie et les grandes privatisations des anciennes entreprises publiques consolidées, incorporant même par la suite les sociétés de distribution d'eau (Durán G., 2015 : 226). Alors même que les chiffres de la croissance sont impressionnants durant la première décennie de la nouvelle démocratie (moyenne de 5 à $7 \%$ entre 1990 et 2000) et que les taux de pauvreté ont considérablement diminué, la population chilienne a dû faire face à un processus de privatisation de la vie sociale dans tous les domaines. L'incertitude et la peur de la précarité se sont installées comme le nouveau sens commun. Cependant, le 
système fonctionnait toujours car la promesse d'un avenir meilleur pour les nouvelles générations restait intacte. On nous disait que l'effort personnel était suffisant pour réussir, et à ces fins l'endettement et la consommation ont été ouverts à de nouveaux groupes de population qui n'avaient jamais eu accès aux biens de qualité. L'augmentation des exportations de cuivre, de cellulose et de produits agricoles a contribué à maintenir les chiffres du chômage relativement bas, tout en faisant entrer des devises étrangères dans le pays. En effet, le Chili s'est enrichi grâce à ces activités extractives, mais la concentration des richesses commençait à prendre des caractéristiques inquiétantes, alors que la plupart de la population a dû faire face à une augmentation croissante du coût de la vie, notamment sur des questions essentielles comme l'éducation, la santé, l'alimentation et le transport.

9 La coalition de centre-gauche au pouvoir a assumé les restrictions démocratiques de la constitution et le veto de la minorité de droite comme le prix à payer pour la stabilité du modèle. Des taux de croissance économique élevés semblaient corroborer l'efficacité du statu quo politique. Cela a généré au sein de la Concertación le débat de 1999 entre les " flagellants » et les « complaisants », ces derniers ayant pris la main et défendu tout ce qui avait été fait depuis 1990 et mis en doute la nécessité de réformes importantes du modèle de développement. C'est précisément au sein de ce groupe de centre-gauche que des liens clé se sont tissés avec les grandes entreprises au travers de postes au sein des conseils d'administration (AFP, ISAPRE, etc.), des contrats dans des sociétés réglementées par l'État, voire des sociétés de conseil en communication et image, entre autres. Ces relations ont consolidé une nouvelle élite qui a facilité une série de consensus et a rendu invisibles les besoins et les difficultés d'une grande partie de la population qui avait vu ses conditions de vie s'améliorer, mais n'avait aucune assurance de les maintenir ou de les projeter vers l'avenir.

L'endettement en tant que mode de vie et la ségrégation urbaine comme manifestation physique des différences sociales ont marqué le contexte de la vie quotidienne de la population chilienne dans les années 1990 et 2000 (González F., $2018: 882$ ). Bien que les chiffres macroéconomiques aient continué de montrer des signes positifs, un malaise croissant s'est peu à peu installé dans la société, dont la participation politique a considérablement diminué depuis 1997. De longues heures de transport pour se rendre au travail, des quartiers périphériques avec de mauvais services, l'endettement croissant des familles, les bas salaires, la criminalité et l'emploi précaire font désormais partie de la vie quotidienne du pays. À cela s'ajoutaient des conflits locaux provoqués par la pollution et la destruction de l'environnement en raison des activités extractives qui dominent l'économie chilienne jusqu'à aujourd'hui. Des régions importantes du pays ont commencé à être abandonnées par l'État, tout comme des services allant de l'éducation aux espaces verts, aux soins de santé primaires et à la protection contre la criminalité. L'économie nationale a continué de croître, mais les fruits de cette croissance ont été répartis de plus en plus inégalement, tandis que l'idée de l'ascenseur social a commencé à s'effondrer. Un nouveau danger se profilait à l'horizon proche : la première grande cohorte de travailleurs du système AFP de capitalisation individuelle prendrait sa retraite vers 2020, ce qui impliquait, selon divers auteurs (Huneeus C., $2018: 2$ ), une baisse significative d'au moins $50 \%$ du revenu pour les nouveaux retraités qui pourrait même être inférieur au salaire minimum. Il n'est donc pas si étrange que le cycle de mobilisation d'octobre 2019 ait coïncidé avec la demande de meilleures pensions. 


\section{Le début du mécontentement}

11 Les bénéfices sociaux de la consommation et du crédit n'ont pas suffi à contenir ce mécontentement qui s'est manifesté entre mai et août 2006 chez les jeunes générations après l'augmentation du prix du transport scolaire. Ce fut l'un des premiers signes massifs du malaise social, bien qu'à l'origine limité aux élèves du secondaire. Les manifestations de 2011 ont marqué une nouvelle étape en raison de leur portée nationale et la durée du mouvement. Celles-ci se sont également déroulées dans le contexte du premier gouvernement de droite qui a remporté des élections démocratiques depuis 1990. Pour la première fois depuis le retour à la démocratie les manifestations ont été dirigées contre tous les acteurs politiques et économiques dans leur ensemble, sans différenciation entre gauche et droite. On a parlé d'une remise en cause générale du modèle économique et de la précarité et de l'endettement qu'il a engendrés pour la plupart des Chiliens. Le problème des inégalités et de la concentration excessive des richesses a pris d'assaut le débat public (Solimano A., 2012 : 25), désignant les principaux acteurs de la transition démocratique comme responsables de la consolidation du modèle et la perte d'intérêt et de légitimité de l'activité politique (Márquez R., 2015 : 86-87). Le sujet de la Constitution de 1980 a émergé dans le débat comme l'un des principaux obstacles aux changements sociaux exigés par la population. Il a également été fait référence à la Cour constitutionnelle (Tribunal Constitucional) en tant qu'organe qui agit par-dessus la légitimité du Parlement et qui fausse la volonté citoyenne. Ainsi, l'idée que la Constitution fonctionnait comme un frein aux revendications sociales et comme un bouclier protecteur du statu quo s'est installée.

Le deuxième gouvernement de Michelle Bachelet (2014-2017) était soutenu par une coalition ( $\mathrm{La}$ nouvelle majorité) qui incorporait d'autres acteurs politiques que la Concertación, tels que le Parti communiste. Son programme de réformes visait à répondre aux demandes du mouvement social de 2011: une éducation gratuite et de qualité ; un processus constituant ; et la réduction des inégalités sociales (Rodríguez J., 2018 : 10). On parlait à l'époque d'un changement de modèle "au sein du modèle " à travers des réformes émanant du pouvoir exécutif et confirmées par le Congrès national. Cependant, les divisions internes de la coalition au pouvoir, l'obstructionnisme de l'opposition de droite et une série de cas de corruption, de collusion et de financement illégal de la politique ont provoqué l'échec d'une grande partie des réformes proposées. L'idée qu'un changement profond du modèle économique était pratiquement impossible, même si l'on détenait le pouvoir exécutif et une majorité parlementaire, s'est installée dans une grande partie de la population. Le problème était alors structurel, car la Cour constitutionnelle, la puissance économique du patronat, la corruption des élites et des lobbies, pouvaient bloquer toute réforme.

L'un des principaux symptômes de ce mécontentement s'est manifesté par l'irruption du mouvement « NO + AFP », qui a rassemblé divers acteurs sociaux opposés au système de retraites créé par la dictature. La demande ne concernait pas seulement les jeunes et les étudiants, mais une masse importante de travailleurs et de personnes âgées qui reprochent à l'État et aux AFP de fournir des pensions atteignant (en moyenne) à peine un tiers des revenus de la vie active. Si l'on ajoute à cela les protestations contre les crises environnementales des soi-disant "zones de sacrifice ", contre la privatisation 
de l'eau, les activités extractives, la mauvaise qualité des transports publics (Araujo K., 2019: 23), et les différents mouvements pour l'accès à un logement décent, un panorama des troubles sociaux généralisés a été mis en place dans la société chilienne ${ }^{2}$. Ce processus peut être apprécié dans les statistiques de l'observatoire des conflits sociaux du $\mathrm{COES}^{3}$, et confirme une hausse soutenue de la contestation sociale dans tout le pays ${ }^{4}$.

Paradoxalement, en 2017, la coalition de droite (dirigée à nouveau par Sebastián Piñera) a remporté les élections présidentielles avec une majorité importante, bien qu'avec un niveau d'abstention record dans l'histoire récente du pays, qui a dépassé $50 \%$ de l'électorat. Une grande partie de ce résultat était due au désenchantement des électeurs de centre-gauche envers le gouvernement Bachelet, qui s'est manifesté par la dispersion des voix entre les différentes listes et le maigre résultat du candidat progressiste au deuxième tour. La lecture de ces résultats par la droite insistait sur la volonté de défaire les réformes promues par le gouvernement de la Nouvelle majorité, en pariant désormais sur le libre marché, la croissance, l'emploi et de nouvelles réformes libérales.

\section{Conclusion}

Après le cycle massif de protestations et de mobilisation sociale initié en octobre 2019, et selon la ligne de notre analyse qui montre un mécontentement croissant envers le modèle économique néolibéral, il est difficile d'imaginer un diagnostic pire que celui mené par la coalition conservatrice en 2017. Aujourd'hui le soutien au président Piñera a atteint un seuil historique proche de $10 \%$, contraignant ce dernier à engager un processus constitutionnel pour 2020 et à promouvoir un agenda de réformes sociales beaucoup plus approfondi que celui proposé par Bachelet en 2013. Cependant, le grand problème de la politique chilienne est précisément l'immense discrédit et la méfiance envers les institutions du pays (y compris la Police, l'Armée et l'Église catholique), qui se reflètent notamment dans le rejet de la classe politique dans son ensemble et des trois pouvoirs de l'État. L'extrême privatisation de la vie imposée au Chili, il y a près de 50 ans, a fini par saper cette confiance, au point de voir s'éroder, de manière inquiétante, l'ensemble du système démocratique.

\section{BIBLIOGRAPHIE}

Araujo, Kathya, « La percepción de las desigualdades : interacciones sociales y procesos sociohistóricos. El caso de Chile », Revista Desacatos, nº 59, enero-abril 2019, p. 16-31.

Durán, Gustavo, « Agua y pobreza en Santiago de Chile. Morfología de la inequidad en la distribución del consumo domiciliario de agua potable », EURE, vol. 41 no 124, 2015, p. 225-246.

Gárate, Manuel, La revolución capitalista de Chile (1973-2003), Santiago, Ediciones Universidad Alberto Hurtado, 2012. 
González López, Felipe, « Crédito, deuda y gubernamentalidad financiera en Chile », Revista Mexicana de Sociología, vol. 80, n.4, 2018, p. 881-908.

Huneeus, Carlos, « La crisis del sistema privado de pensiones : un punto de inflexión de nuestra democracia », Ciper Chile, Santiago, 30 août 2018, https://urlz.fr/bBjL, page consultée le $09 / 03 / 2020$

Márquez, Rodrigo, « La distancia entre la ciudadanía y las élites. Una mirada desde el informe Desarrollo humano en Chile », Estudios Públicos, nº 40, 2015, p. 83-106.

Milos, Pedro, Historia y memoria : 2 de abril de 1957, Santiago, Ediciones LOM, 2007.

Palma, Gabriel, « Chile 1914-1935 : de economía exportadora a sustituidora de importaciones », Colección estudios CIEPLAN, n ${ }^{\circ}$, mars 1984, p. 61-88.

Rodríguez, Javier, Desarrollo y desigualdad en Chile (1850-2009) : Historia de su economía política, Santiago, LOM Ediciones, 2018.

Solimano, Andrés, Capitalismo a la chilena, Santiago, Editorial Catalonia, 2012.

Valdivia, Verónica, El golpe después del golpe : Leigh vs. Pinochet, Chile 1960-1980, Santiago, LOM ediciones, 2003.

\section{NOTES}

1. «Institutions de santé prévisionnelle " et "Associations des fonds de pensions" respectivement.

2. Le rapport du PNUD (Programme des Nations Unies pour le développement) pour 2017, intitulé «Desiguales» (Inégaux), a clairement montré l'inégalité économique dans le pays malgré les bons indices macroéconomiques. Ce rapport a mis un accent particulier sur la question des abus en général, de la dignité des personnes et de la ségrégation économique. Voir : www.cl.undp.org 3. Centre d'études sur les conflits et la cohésion sociale.

4. Voir le site de l'Observatoire des conflits sociaux du COES: https://coes.cl/observatorio-deconflicto/

\section{AUTEUR}

\section{MANUEL GÁRATE}

Manuel Gárate est affilié au COES / Université catholique du Chile mgarate@uc.cl 